\title{
An Open-Source Implementation of Analytical Turbulence-Airfoil Interaction Noise Model
}

\author{
F. Casagrande Hirono* \\ P. F. Joseph ${ }^{\dagger}$ \\ F. M. Fazi ${ }^{\ddagger}$ \\ Institute of Sound and Vibration Research, University of Southampton, Southampton, UK, SO17 1BJ.
}

\begin{abstract}
An open-source implementation of the Amiet model for turbulence-airfoil interaction noise implemented in Python programming language is presented. The proposed implementation, named amiet_tools, allows the user to calculate the unsteady surface pressure jump and the radiated acoustic pressure due to a single or multiple turbulent gusts interacting with a flat plate airfoil. Secondary effects such as the acoustic near-field, finite-span effects, subcritical gusts contribution, and sound refraction at a planar shear layer are available for simulating a realistic open-jet wind tunnel test setup. Prediction results are presented and compared to measured acoustic pressures obtained in an open-jet wind tunnel with a planar spiral microphone array, and good agreement is obtained over a wide range of frequencies. A link for downloading the package codes and sample experimental data is included. It is expected the proposed implementation can be adopted as a quick benchmark for more advanced analytical airfoil noise models and experimental measurements.
\end{abstract}

\section{Introduction}

Noise due to incoming turbulence interacting with a lifting surface is a fundamental source of broadband noise in the aviation industry. While its fundamental mechanisms are fairly well understood, efficient methods for airfoil noise reduction are still subject of ongoing research. Recent years have seen significant advances in airfoil geometry modifications for noise reductions based on combinations of analytical, ${ }^{1,2}$ numerical ${ }^{3}$ and experimental research work. ${ }^{4-8}$ While analytical models generally require significant simplifications and assumptions about the problem geometry and flow properties, they provide fast and cheap results that are well suited for studying scaling laws and the underlying physics. ${ }^{9}$

Much of the current research in airfoil noise originated from seminal work developed in the 1970s by Adamczyk, ${ }^{10}$ Graham, ${ }^{11}$ Amiet, ${ }^{12}$ amongst many others. ${ }^{9}$ Amongst these, Amiet's paper from $1975^{12}$ is often considered one of the most important references for analytical models of leading edge noise, particularly due to the formal simplicity of his approach and range of extensions it offers. Indeed, extensions to this model have been investigated and proposed in recent years. ${ }^{13,14}$ The same analytical framework has been adopted by Amiet to develop a similar model for trailing edge noise, which is also often adopted in the literature. ${ }^{15}$

In short, Amiet's model decomposes the incoming turbulent flow into a incoherent sum of sinusoidal gusts, and relates the acoustic power spectral density seen by an observer in the far-field directly to the incoming flow properties and to the airfoil geometry. In particular, under an infinite-span approximation, it is shown that only a single sinusoidal gust will be responsible for all the noise seen by the far-field observer. ${ }^{15}$ This expression bypasses the need to calculate the acoustic source distribution over the airfoil surface and to compute the full radiation integral, and is often sufficient for obtaining general trends of the radiated noise.

However, more advanced problems might not allow such approximations to be made, and instead require calculations of the radiated acoustic near-field or the inclusion of finite span effects. Under these conditions, the Amiet model requires the separate calculation of the acoustic source distribution and of the acoustic radiation, becoming significantly more complex to implement. Researchers might also be interested in investigating the flat plate surface

*Postgraduate Research Student, fchirono@gmail.com, Member AIAA

${ }^{\dagger}$ Professor, pf j@isvr.soton.ac.uk, Member AIAA

‡Associate Professor, filippo.fazi@soton.ac.uk 
pressure characteristics predicted by the Amiet model, in an effort to better understand the acoustic source properties and develop more efficient noise reduction techniques at the source. In either of the above mentioned cases, a reference implementation of the Amiet model would allow researchers to quickly obtain predictions of leading edge noise via a well-established model and avoid spending time with implementation details, allowing them to focus on developing novel extensions or validating experimental results.

The use of an open-source package by a community incentivises users to reuse code instead of rewriting code, which is considered a good practice to reduce errors when developing scientific software. ${ }^{16}$ Possible errors in its implementation are more likely to be found and reported back to the developers if the software is accessible by a larger number of users, thus hopefully improving it with time. Recent examples of open-source software implementations in the field of aeroacoustics are the Acoular ${ }^{17}$ package for microphone array data processing, and the noisyduck ${ }^{18}$ tool for computing eigenmode decompositions of duct flows, both using the Python programming language.

This work presents an open-source implementation of the Amiet model for leading-edge noise. In order to make it available for a large number of researchers, the implementation is made in Python programming language, which is freely available and works across multiple operating systems. The package is published under a permissible open license, which allows the distribution, modification, and private use of the codes. Users are invited to contribute to the code development with suggestions and requests at the project web page or by contacting the first author. The implementation also serves a didactic purpose, by demonstrating the steps in Amiet's flat plate theory to calculate the radiated noise.

The package codes, together with a few example Python scripts and two microphone array cross-spectral matrix files (one containing analytical results using the model, and one containing measurements performed in equivalent conditions), are freely available from the link below:

- https://github.com/fchirono/amiet_tools

In the following sections, we will briefly describe the main concepts of the Amiet model for leading edge noise, followed by a description of its numerical implementation and some of the decisions taken throughout its development. We then describe a set of experiments conducted in the ISVR open-jet wind tunnel using a flat-plate airfoil and a planar microphone array, and compare the predicted and the experimental results for the measured microphone signals in terms of acoustic power spectral density magnitude, cross-spectral phase and coherence. Finally, Appendix A describes the algorithm pseudocode, and Appendix B describes the Python classes used to input data to the code.

\section{Airfoil Response to a Turbulent Gust}

\section{II.A. Airfoil Surface Pressure}

The classical problem of a flat plate interacting with an isotropic turbulent flow has been extensively discussed in the literature, and therefore only a brief description of its main features is given below. For more in-depth analyses, see references ${ }^{15,19-21}$, for example.

The problem setup is shown in Figure 1: a flat plate airfoil is immersed in a turbulent, subsonic moving medium flowing with Mach number $M_{x}=U_{x} / c_{0}$ in the $+x$ direction. The airfoil has chord $c=2 b$ and $\operatorname{span} L=2 d$, and the origin of the coordinate system is placed at the center of the airfoil, with the positive $z$ coordinate pointing upwards ("out of the page" in Fig. 1). We denote points in the space around the airfoil as $\mathbf{r}=(x, y, z)$, and points on the airfoil surface with a subscript " " ", as $\mathbf{r}_{s}=\left(x_{s}, y_{s}, z_{s}=0\right)$.

A continuum of "frozen" sinusoidal turbulent gusts move in the plane $z=0$ at the convection velocity $U_{x}$, and each gust is denoted by a pair of streamwise and spanwise hydrodynamic (i.e. vortical) wavenumbers $\left(k_{\chi}, k_{\psi}\right)$. Leaving the time dependence $e^{+j \omega t}$ implicit, a surface pressure jump $\Delta p$ will develop over the airfoil surface in response to the incoming turbulence of the form

$$
\Delta p\left(x_{s}, y_{s}, \omega\right)=2 \pi \rho_{0} \int_{-\infty}^{+\infty} w\left(\kappa_{\chi}, k_{\psi}\right) g\left(x_{s}, \kappa_{\chi}, k_{\psi}\right) e^{-j k_{\psi} y_{s}} \mathrm{~d} k_{\psi},
$$

where $w\left(k_{\chi}, k_{\psi}\right)$ is the gust amplitude, $\kappa_{\chi}=\omega / U_{x}$ is the gust chordwise wavenumber at frequency $\omega=2 \pi f$, and $g$ is the the non-dimensional pressure jump over the chord $x_{s}$ due to each gust component $\left(\kappa_{\chi}, k_{\psi}\right)$.

However, turbulence is a random phenomenon, and must be analysed in a statistical sense. The cross-power spectral density between the surface pressure jump at two points $\left(x_{s}, y_{s}\right)$ and $\left(x_{s}^{\prime}, y_{s}^{\prime}\right)$ can be defined as 


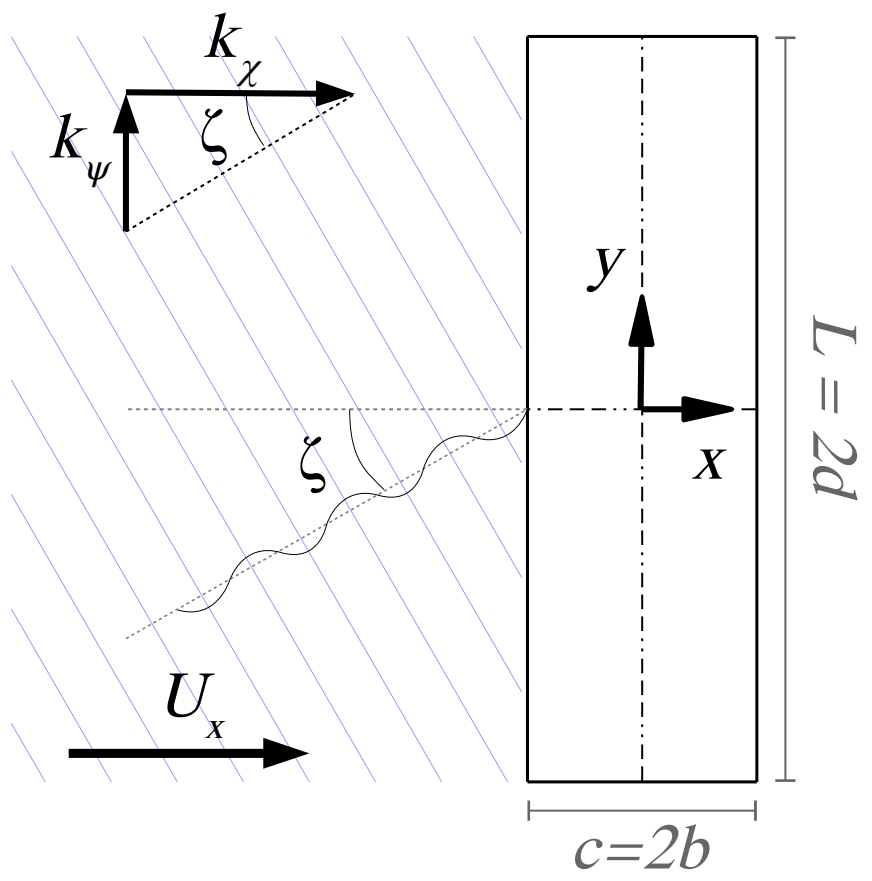

Figure 1: Experimental setup diagram, with gust incidence angle $\zeta=\arctan \left(k_{\psi} / k_{\chi}\right)$.

$$
\begin{aligned}
S_{\Delta p \Delta p^{\prime}}\left(x_{s}, x_{s}^{\prime}, y_{s}, y_{s}^{\prime}, \omega\right) & =\lim _{T \rightarrow \infty}\left[\frac{\pi}{T} \mathrm{E}\left\{\Delta p\left(x_{s}, y_{s}, \omega\right) \Delta p^{*}\left(x_{s}^{\prime}, y_{s}^{\prime}, \omega\right)\right\}\right] \\
& =\left(2 \pi \rho_{0}\right)^{2} U_{x} \int_{-\infty}^{+\infty} \Phi_{w w}\left(\kappa_{\chi}, k_{\psi}\right) g\left(x_{s}, \kappa_{\chi}, k_{\psi}\right) g^{*}\left(x_{s}^{\prime}, \kappa_{\chi}, k_{\psi}\right) e^{-j k_{\psi}\left(y_{s}-y_{s}^{\prime}\right)} \mathrm{d} k_{\psi}
\end{aligned}
$$

where $\Phi_{w w}\left(k_{\chi}, k_{\psi}\right)$ is the incoming turbulence wavenumber spectral density.

\section{II.B. Airfoil Acoustic Radiation}

The acoustic pressure radiated by the airfoil can be obtained from the Ffowcs-Williams and Hawkings acoustic analogy. ${ }^{22}$ The total radiated acoustic pressure $p(\mathbf{r}, \omega)$ seen by an observer located at $\mathbf{r}=(x, y, z)$ is obtained by integrating the surface pressure jump, which is interpreted as the amplitude distribution of point dipole sources normal to the flat plate:

$$
p(\mathbf{r}, \omega)=\int_{-d}^{d} \int_{-b}^{b} \Delta p\left(x_{s}, y_{s}, \omega\right) \frac{\partial}{\partial z_{s}} G_{U_{x}}\left(\mathbf{r} \mid \mathbf{r}_{s}, \omega\right) \mathrm{d} x_{s} \mathrm{~d} y_{s},
$$

where $\partial G_{U_{x}} / \partial z_{s}$ is the transfer function for a point dipole source in a convected medium:

$$
\frac{\partial}{\partial z_{s}} G_{U_{x}}\left(\mathbf{r} \mid \mathbf{r}_{s}, \omega\right)=\left(j k_{0}+\frac{1}{\bar{r}}\right) \frac{\left(\bar{z}-\bar{z}_{s}\right)}{\beta \bar{r}} \frac{e^{-j k_{0} \bar{r}}}{4 \pi \beta^{2} \bar{r}} e^{j k_{0} M_{x}\left(\overline{\bar{x}}-\overline{\bar{x}}_{s}\right)},
$$

and the overline represents flow-transformed variables: ${ }^{23}$

$$
\overline{\mathbf{r}}=(\overline{\bar{x}}, \bar{y}, \bar{z})=\left(\frac{x}{\beta^{2}}, \frac{y}{\beta}, \frac{z}{\beta}\right), \quad \bar{r}=\left\|\overline{\mathbf{r}}-\overline{\mathbf{r}}_{s}\right\|, \quad \beta=\sqrt{1-M_{x}^{2}} .
$$

The cross-power spectral density $S_{p p^{\prime}}\left(\mathbf{r}, \mathbf{r}^{\prime}, \omega\right)$ between the acoustic pressure at two observer points $\mathbf{r}$ and $\mathbf{r}^{\prime}$ is: 


$$
\begin{aligned}
S_{p p^{\prime}}\left(\mathbf{r}, \mathbf{r}^{\prime}, \omega\right) & =\lim _{T \rightarrow \infty}\left[\frac{\pi}{T} \mathrm{E}\left\{p(\mathbf{r}, \omega) p^{*}\left(\mathbf{r}^{\prime}, \omega\right)\right\}\right] \\
& =\int_{-d}^{+d} \int_{-b}^{+b} \int_{-d}^{+d} \int_{-b}^{+b} S_{\Delta p \Delta p^{\prime}}\left(\mathbf{r}_{s}, \mathbf{r}_{s}^{\prime}, \omega\right) \frac{\partial}{\partial z_{s}} G_{U_{x}}\left(\mathbf{r} \mid \mathbf{r}_{s}, \omega\right) \frac{\partial}{\partial z_{s}^{\prime}} G_{U_{x}}^{*}\left(\mathbf{r}^{\prime} \mid \mathbf{r}_{s}^{\prime}, \omega\right) \mathrm{d} x_{s} \mathrm{~d} y_{s} \mathrm{~d} x_{s}^{\prime} \mathrm{d} y_{s}^{\prime} .
\end{aligned}
$$

Equation 8 is the most general airfoil-turbulence interaction noise model, and includes near-field effects and the effects of subcritical gusts (discussed below). However, it does not lend itself to obtaining closed-form analytical solutions, nor does it yield any insight into the acoustic radiation of the airfoil.

\section{II.C. Far-Field Approximation}

In the original paper, Amiet ${ }^{12}$ simplified Equation 8 using a far-field approximation. When the observer is in the geometric far-field of the source, Eq. 5 can be approximated as

$$
\frac{\partial}{\partial z_{s}} G_{F F}\left(\mathbf{r} \mid \mathbf{r}_{s}, \omega\right)=\frac{j k_{0} z}{4 \pi \sigma_{\mathbf{r}}^{2}} e^{j k_{0}\left(\frac{M_{x} x-\sigma_{\mathbf{r}}}{\beta^{2}}\right)} e^{j k_{0}\left(\frac{x-M_{x} \sigma_{\mathbf{r}}}{\sigma_{\mathbf{r}} \beta^{2}}\right) x_{s}} e^{j k_{0}\left(\frac{y}{\sigma_{\mathbf{r}}}\right) y_{s}},
$$

where $\sigma_{\mathbf{r}}$ is the flow-corrected distance from the observer to the source centre, given by

$$
\sigma_{\mathbf{r}}=\sqrt{x^{2}+\beta^{2}\left(y^{2}+z^{2}\right)}=\beta^{2}\|\overline{\mathbf{r}}\|
$$

\section{II.C.1. Far-Field, Finite Span Formulation}

By adopting the far-field approximation for the dipole acoustic radiation, it is possible to simplify the radiation integral (Eq. 4) and separate the chordwise from the spanwise integration:

$$
\begin{gathered}
p(\mathbf{r}, \omega) \approx \int_{-d}^{d} \int_{-b}^{b}\left[2 \pi \rho_{0} \int_{-\infty}^{+\infty} w\left(\kappa_{\chi}, k_{\psi}\right) g\left(x_{s}, \kappa_{\chi}, k_{\psi}\right) e^{-j k_{\psi} y_{s}} \mathrm{~d} k_{\psi}\right] \ldots \\
\ldots\left[\frac{j k_{0} z}{4 \pi \sigma_{\mathbf{r}}^{2}} e^{j k_{0}\left(\frac{M_{\chi} x-\sigma_{\mathbf{r}}}{\beta^{2}}\right)} e^{j k_{0}\left(\frac{x-M_{\chi} \sigma_{\mathbf{r}}}{\sigma_{\mathbf{r}} \beta^{2}}\right) x_{s}} e^{j k_{0}\left(\frac{y}{\sigma_{\mathbf{r}}}\right) y_{s}}\right] \mathrm{d} x_{s} \mathrm{~d} y_{s} \\
=\rho_{0} \frac{j k_{0} b z}{2 \sigma_{\mathbf{r}}^{2}} e^{j k_{0}\left(\frac{M_{\chi} x-\sigma_{\mathbf{r}}}{\beta^{2}}\right)} \int_{-\infty}^{+\infty} w\left(\kappa_{\chi}, k_{\psi}\right) \mathscr{L}\left(x, \kappa_{\chi}, k_{\psi}\right) \frac{(2 d) \sin \left(\left(k_{\psi}-\kappa_{\psi}\right) d\right)}{\left(k_{\psi}-\kappa_{\psi}\right) d} \mathrm{~d} k_{\psi},
\end{gathered}
$$

where $\kappa_{\psi}=k_{0} y / \sigma_{\mathbf{r}}$, and $\mathscr{L}\left(x, k_{\chi}, k_{\psi}\right)$ is the effective lift ${ }^{24}$ for a single turbulent gust $\left(k_{\chi}, k_{\psi}\right)$ :

$$
\mathscr{L}\left(x, k_{\chi}, k_{\psi}\right)=\frac{1}{b} \int_{-b}^{b} g\left(x_{s}, k_{\chi}, k_{\psi}\right) e^{j k_{0}\left(\frac{x-M_{\chi} \sigma_{\mathrm{r}}}{\sigma_{\mathrm{r}} \beta^{2}}\right) x_{s}} \mathrm{~d} x_{s} .
$$

The effective lift function in Eq. 13 describes the airfoil acoustic directivity over the chordwise angles for a single gust with wavenumbers $\left(k_{\chi}, k_{\psi}\right)$, as its implicit dependence on the flow-corrected chordwise observer angle $x / \sigma_{\mathbf{r}}$ indicates. There are known closed-form solutions available for the effective lift function., ${ }^{9}, 19$

Similarly, the spanwise integration of the airfoil noise results in a sinc function in $k_{\psi}$-domain, centred at $\kappa_{\psi}=$ $k_{0} y / \sigma_{\mathbf{r}}$. This function depends on the flow-corrected observer spanwise angle $y / \sigma_{\mathbf{r}}$, and as such denotes the airfoil acoustic directivity over the spanwise angles for a single gust with wavenumbers $\left(k_{\chi}, k_{\psi}\right)$. For example, an observer located at mid-span $(y=0)$ will be exposed mostly to the acoustic radiation induced by the gust with spanwise wavenumber $k_{\psi}=0$, and will observe a much smaller contribution from oblique $\left(k_{\psi} \neq 0\right)$ gusts.

The width of the sinc function main lobe decreases for increasing frequency and/or airfoil span, leading to a narrower spanwise directivity under these conditions - and likewise, to a broader directivity at low frequencies and/or short span. As the gust components in isotropic turbulence are assumed incoherent, observers positioned at different spanwise angles will observe a variable degree of coherence across them, depending on whether they are "illuminated" by a single or by multiple gusts. This tendency is verified in Section $\mathrm{V}$ for both predicted and experimental acoustic measurements across different spanwise angles.

The acoustic power spectral density of the far-field sound $S_{p p}(\mathbf{r}, \omega)$ can be written as

$$
S_{p p}(\mathbf{r}, \omega)=\left(\frac{\rho_{0} k_{0} b z}{2 \sigma_{\mathbf{r}}^{2}}\right)^{2} U_{x} \int_{-\infty}^{+\infty} \Phi_{w w}\left(\kappa_{\chi}, k_{\psi}\right)\left\|\mathscr{L}\left(x, \kappa_{\chi}, k_{\psi}\right)\right\|^{2}\left\|\frac{(2 d) \sin \left(\left(k_{\psi}-\kappa_{\psi}\right) d\right)}{\left(k_{\psi}-\kappa_{\psi}\right) d}\right\|^{2} \mathrm{~d} k_{\psi} .
$$




\section{II.C.2. Far-Field, Infinite Span Formulation}

In the limit of the airfoil span becoming large (i.e. $d \rightarrow \infty$ ), the sinc function obtained above will tend to behave as a Dirac Delta function in $k_{\psi}$. The integration over the $k_{\psi}$ wavenumbers coupled with the Delta function will effectively sift the spanwise turbulence wavenumber, so that only the gust with wavenumber $\kappa_{\psi}=k_{0} y / \sigma_{\mathbf{r}}$ will contribute to the sound observed at the spanwise direction $y / \sigma_{\mathbf{r}}$ in the far field. For an observer at mid-span $(y=0)$, only the normal incidence gust $k_{\psi}=0$ will contribute to the observed acoustic pressure, while all oblique gusts $\left(k_{\psi} \neq 0\right)$ will cancel along the span and will not radiate towards this observer.

The far field, infinite-span pressure can be approximated by the acoustic field generated by a single gust with hydrodynamic wavenumbers $\left(\kappa_{\chi}, \kappa_{\psi}\right)$ :

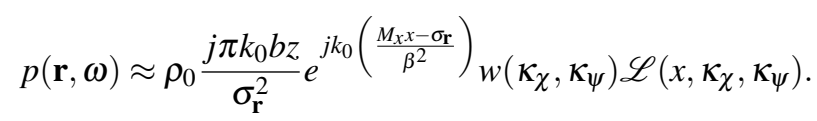

The far-field, infinite-span power spectral density $S_{p p}(\mathbf{r}, \omega)$ can be written as:

$$
S_{p p}(\mathbf{r}, \omega)=\left(\frac{\rho_{0} k_{0} b z}{\sigma_{\mathbf{r}}^{2}}\right)^{2} U_{x} \pi d \Phi_{w w}\left(\kappa_{\chi}, \kappa_{\psi}\right)\left\|\mathscr{L}\left(x, \kappa_{\chi}, \kappa_{\psi}\right)\right\|^{2} .
$$

Equation 16 is the more popular form of the Amiet model for airfoil-interaction noise, as it offers a direct relationship between the problem parameters - namely, the airfoil geometry and flow parameters - to the noise seen by a distant observer.

\section{II.D. Supercritical and Subcritical Gusts}

The proposed implementation of Amiet's interaction noise model requires calculating the airfoil response to a range of discrete gusts. Graham ${ }^{11}$ described how the response of an infinite-span airfoil subject to a subsonic, oblique gust can assume two forms, depending on whether the spanwise gust wavenumber $k_{\psi}$ is above or below a critical spanwise wavenumber $k_{\psi}^{\text {crit }}$, given by

$$
k_{\psi}^{c r i t}=\frac{k_{\chi} M_{x}}{\beta} .
$$

A gust is termed supercritical when $\left|k_{\psi}\right|<k_{\psi}^{\text {crit }}$. Such gusts will induce a surface pressure jump that is distributed over a large section of the airfoil chord, ${ }^{9}$ and the airfoil response will be an efficient radiator of sound to the far-field.

A gust is termed subcritical when $\left|k_{\psi}\right|>k_{\psi}^{\text {crit }}$. These gusts will induce a surface pressure jump that decays exponentially from the leading edge towards the trailing edge; moreover, acoustic radiation from adjacent regions will cancel along the span, and the airfoil will be an inefficient radiator of far-field sound. ${ }^{9}$ However, subcritical gusts are significant sources of far-field sound at low frequencies due to incomplete cancellation effects along the span., ${ }^{9,21}$

The airfoil response function $g\left(x_{s}, k_{\chi}, k_{\psi}\right)$ assumes different forms for each type of gusts; see, for example, Reboul ${ }^{19}$ or de Santana ${ }^{20}$ for formulations. In order to obtain a smooth transition around criticality, the airfoil response to a critical gust $\left(\left|k_{\psi}\right|=k_{\psi}^{\text {crit }}\right)$ is obtained from numerical interpolation between responses slightly above and slightly below $k_{\psi}^{\text {crit }}$, as suggested in Roger. ${ }^{9}$

When not assuming far-field conditions, it is generally necessary to consider the contributions of both supercritical $\left(\left|k_{\psi}\right|<k_{\psi}^{c r i t}\right)$ and subcritical gusts $\left(\left|k_{\psi}\right|>k_{\psi}^{c r i t}\right)$ to the surface pressure cross-spectral density in Eq. 3. However, the gusts' contribution to the surface pressure tend to decrease as $\left|k_{\psi}\right|$ increases, and the infinite integration in Eq. 3 can be well approximated by a finite, discrete summation up to a maximum spanwise gust wavenumber $k_{\psi}^{\max }$ of interest. Section III.B discusses criteria for determining this limit.

\section{II.E. Turbulent Velocity Wavenumber Spectrum}

In order to match the experimental conditions, we adopted the von Karman wavenumber energy spectrum ${ }^{12}$ for homogeneous, isotropic turbulence. This model describes the velocity spectrum vertical to the flat plate $\Phi_{w w}\left(k_{\chi}, k_{\psi}\right)$ as a function of hydrodynamic wavenumbers $\left(k_{\chi}, k_{\psi}\right)$, and is defined in terms of the turbulence mean-squared velocity $\overline{w^{2}}$ and the integral length scale $\Lambda$ as 


$$
\begin{aligned}
\Phi_{w w}\left(k_{\chi}, k_{\psi}\right) & =\frac{4}{9 \pi} \frac{\overline{w^{2}}}{k_{e}^{2}} \frac{\check{k}_{\chi}^{2}+\check{k}_{\psi}^{2}}{\left(1+\check{k}_{\chi}^{2}+\check{k}_{\psi}^{2}\right)^{7 / 3}}, \\
k_{e} & =\frac{\sqrt{\pi}}{\Lambda} \frac{\Gamma(5 / 6)}{\Gamma(1 / 3)},
\end{aligned}
$$

where $\breve{k}_{i}=k_{i} / k_{e}$ and $\Gamma(\cdot)$ is the Gamma function.

\section{Numerical Implementation}

This section covers numerical implementation aspects of the Amiet model presented above, and discusses strategies and decisions adopted during the model implementation. We focus on discussing the general model presented in Eq. 8 , which includes near-field, finite-span and subcritical gusts' effects. The previously mentioned far-field, infinite-span approximation is also included in the amiet_tools package, but is not further discussed due to its relative simplicity.

\section{III.A. Airfoil Surface Discretization}

The non-dimensional chordwise pressure jump function $g$ is known to exhibit a $\left(x_{s}^{-1 / 2}\right)$-type singularity at the leading edge, ${ }^{19,20}$ and integrating the airfoil surface pressure near this singularity demands some extra care. A non-uniform discretisation scheme is adopted in the chordwise direction to deal with this singularity, similar to the method used by Clair et al. ${ }^{4}$ The chord sampling points $x_{s}[n] \in(-b, b]$ are calculated as

$$
x_{S}[n]=\left(\frac{e^{l[n]}-e^{-L_{\text {exp }} / 2}}{e^{+L_{\text {exp }} / 2}-e^{-L_{\exp } / 2}}\right)(2 b)-b,
$$

where $l[n]$ contains $N_{x}+1$ points uniformly sampled over the non-dimensional interval $\left[-L_{\exp } / 2, L_{\text {exp }} / 2\right]$. The first sample of $x_{S}[n]$, located exactly at the leading edge, is then removed from the grid. This exponential function provides a finer discretisation near the leading edge when evaluated over the negative values around $-L_{\text {exp }} / 2$. Shorter interval lengths $L_{\text {exp }}$ provide a more uniform sampling, while larger values provide a more non-uniform sampling. The default value is $L_{\text {exp }}=2$.

The spanwise sampling is uniform, with $N_{y}$ points in the range $y_{s} \in[-d, d]$. Note that the spanwise sampling interval $\Delta y_{s}$ is identical over the entire span, while the chordwise sampling interval $\Delta x_{s}$ is non-uniform and becomes smaller closer to the leading edge. Care must be taken when numerically integrating the surface pressure to consider the correct corresponding sample width.

Figure 2 shows an example of the proposed discretisation with $\left(N_{x}, N_{y}\right)=(50,101)$ points for illustration purposes. A denser grid of $\left(N_{x}, N_{y}\right)=(100,101)$ is suggested for the actual calculations to obtain convergence at higher frequencies.

\section{III.B. Gust Integration Limits}

\section{III.B.1. Surface Pressure Calculations}

Due to their exponential decay towards the trailing edge, subcritical gusts will only affect regions on the airfoil surface near the leading edge, and cease to affect regions that are further downstream. For a given chord point $x_{s}$ on the airfoil surface, there will be a unique subcritical gust, denoted by its spanwise wavenumber $k_{\psi}^{[A]}$, such that spanwise wavenumbers $\left|k_{\psi}\right|<k_{\psi}^{[A]}$ will contribute significantly to the surface pressure response at that point, while gusts with wavenumbers $\left|k_{\psi}\right|>k_{\psi}^{[A]}$ will have their surface pressure contributions at that point attenuated $A$ decibels or more due to their exponential decay, and will be negligible contributors to the surface pressure. This gust wavenumber $k_{\psi}^{[A]}$ will depend on the chord reference point $x_{s}$, Mach number $M_{x}$ and frequency $k_{0}$, and can be determined from the airfoil response function $g$ for subcritical gusts ${ }^{21}$ as

$$
k_{\psi}^{[A]}\left(x_{s}, M_{x}, k_{0}\right)=k_{\psi}^{c r i t} \sqrt{\left[-\frac{\beta^{2} \ln \left(10^{\frac{A}{20}}\right)}{k_{0}\left(x_{s}+b\right)}\right]^{2}+1} .
$$




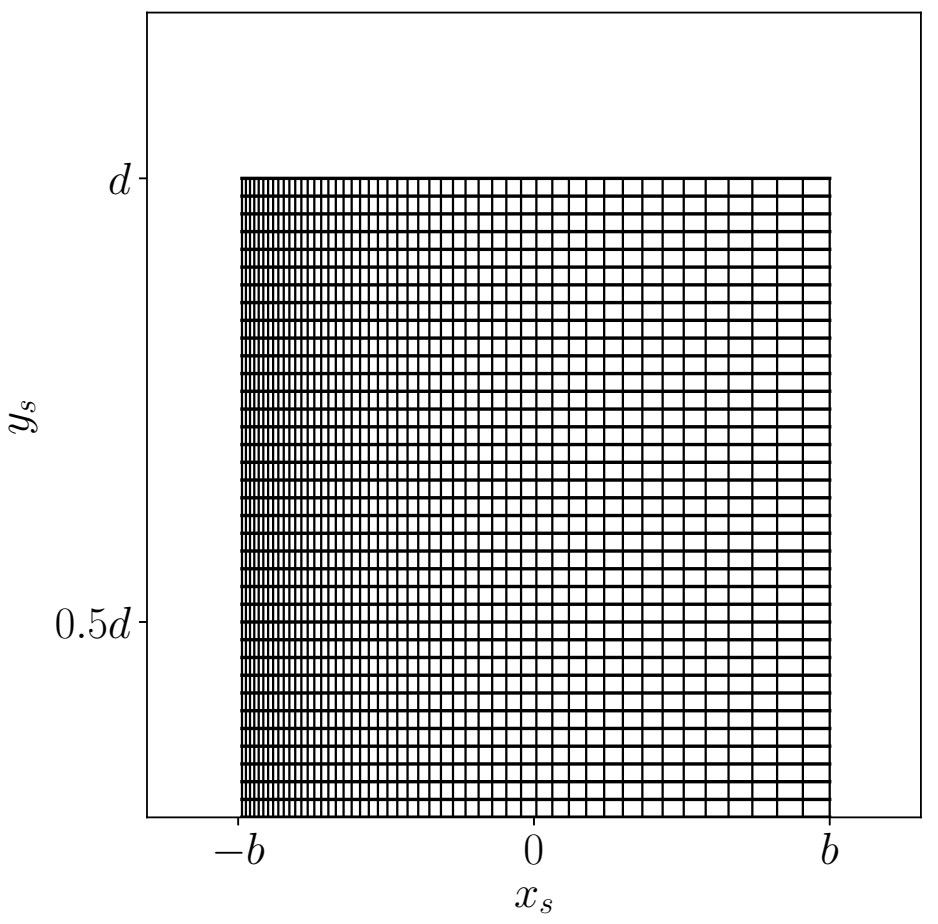

Figure 2: Example of flat-plate airfoil surface discretisation mesh around the span tip, with $N_{y}=101$ points uniformly distributed in the spanwise direction and $N_{x}=50$ points non-uniformly distributed in the chordwise direction.

Figure 3 shows the maximum subcritical gust spanwise wavenumber $k_{\psi}^{[A]}$ for $A=-20 \mathrm{~dB}$ as a function of $x_{s}$ for three chordwise reduced frequencies $k_{0} c=[0.5,5,20]$. It can be seen that as $x_{s}$ moves downstream, the less the airfoil response is affected by subcritical gusts, which can then be ignored in the analysis. Conversely, if $x_{s}$ moves upstream towards the leading edge or if one predicts the airfoil response at lower frequencies, then it is necessary to consider a much larger range of subcritical gusts in order to obtain convergence in the airfoil surface pressure cross-spectral density.

Although not shown here for brevity reasons, the convergence of the surface pressure cross-spectral density $S_{\Delta p \Delta p^{\prime}}\left(\mathbf{r}_{s}, \mathbf{r}_{s}^{\prime}, \omega\right)$ as a function of maximum spanwise gust integration has been investigated, and it was found that $k_{\psi}^{\max }=k_{\psi}^{[-20]}$ (i.e. $A=-20 \mathrm{~dB}$ ) is a sufficient integration limit to achieve convergence in the cross-spectral density for a wide range of frequencies and pairs of points on the flat plate surface. This limit is adopted by default when computing the airfoil surface pressure response to multiple gusts, but can be changed if so desired.

\section{III.B.2. Acoustic Pressure Calculations}

In order to compute the airfoil acoustic radiation, it is not necessary to integrate the contributions of gusts up to the previously proposed wavenumber limit $k_{\psi}^{[-20]}$. As this value generally assumes values well above the critical gust $k_{\psi}^{c r i t}$ for most conditions, it can be excessive for acoustic radiation predictions, where mostly supercritical gusts will be relevant.

It has been previously stated that subcritical gusts are significant contributors to the acoustic radiation at low frequencies, but cease to contribute at higher frequencies. We can then consider a critical frequency $f_{\text {crit }}$ separating both frequency ranges, and investigate criteria for establishing $f_{\text {crit }}$.

In previous sections, the flat plate was shown to have a sinc function-like directivity as a function of gust spanwise wavenumber $k_{\psi}$, as seen in Eq. 14. The isolated sinc function term $\sin \left(k_{\psi} d\right) /\left(k_{\psi} d\right)$ is shown in Figure 4 for an observer at mid-span, where it is shown that the sinc function first and second sidelobe peaks are approximately -13 $\mathrm{dB}$ and $-18 \mathrm{~dB}$ below the main lobe peak.

We propose that gusts in the range $k_{\psi} \in[-2 \pi / d,+2 \pi / d]$ - that is, located within the sinc function main lobe and first sidelobes for an observer at mid-span, and indicated by the shaded region in Figure 4 - will be significant 


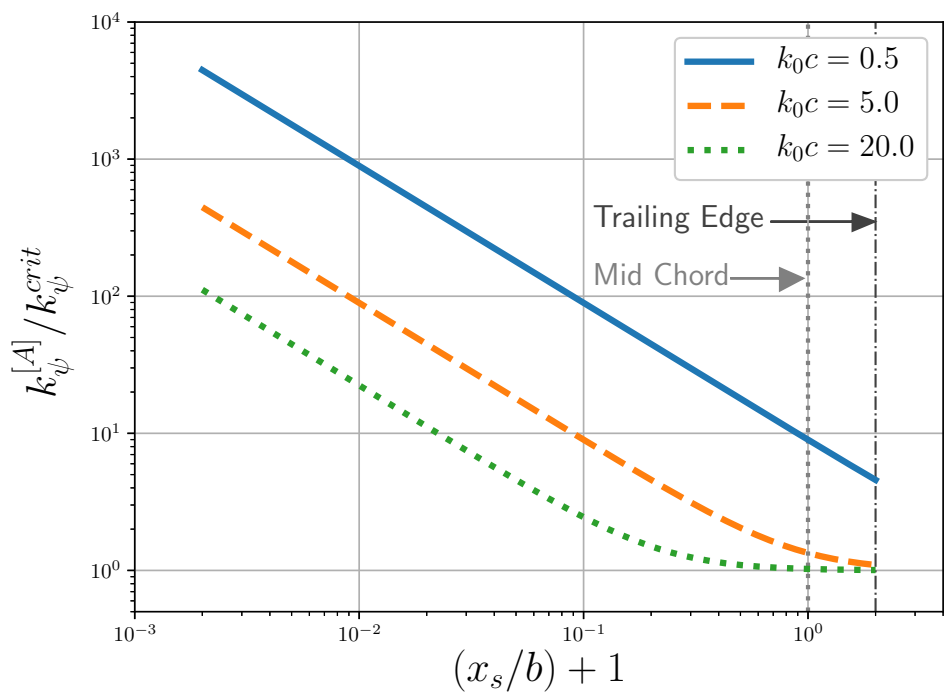

Figure 3: Evanescent gust spanwise wavenumber $k_{\psi}^{[A]}$, with evanescent response at $A=-20 \mathrm{~dB}$ at each chord point $x_{s}$, in double-logarithmic plot, for chordwise normalised frequencies $k_{0} c=[0.5,5,20]$.

contributors to the acoustic field at all frequencies, even when considered subcritical. However, it was also established that gusts in the supercritical range $k_{\psi} \in\left[-k_{\psi}^{c r i t},+k_{\psi}^{c r i t}\right]$ are also significant contributors of far-field sound, although this range changes with frequency. The transition frequency $f_{\text {crit }}$ can thus be determined by comparing the two proposed ranges:

- In the low frequency region, $k_{\psi}^{\text {crit }}<2 \pi / d$, and both supercritical and some subcritical gusts will be efficient radiators of far-field sound. The gust wavenumber integration should include both, and the proposed integration limit is $k_{\psi}^{\max }=2 \pi / d$;

- In the high frequency region, $k_{\psi}^{c r i t} \geq 2 \pi / d$. The sinc function main lobe and first sidelobes are already included within the range of supercritical gusts, and the proposed integration limit is $k_{\psi}^{\max }=k_{\psi}^{c r i t}$.

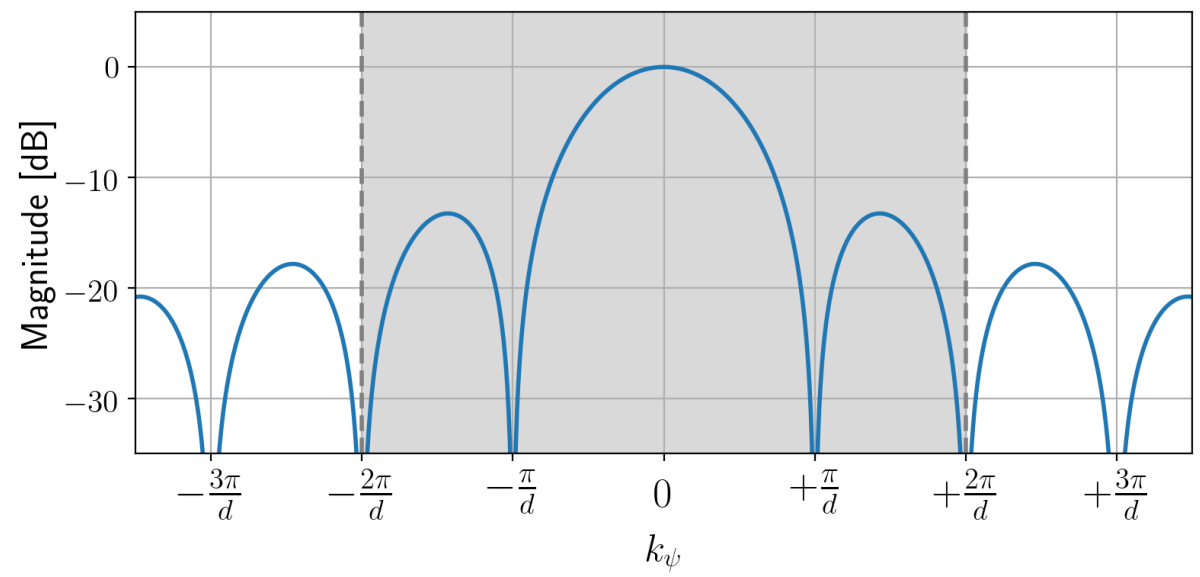

Figure 4: Spanwise directivity function $\sin \left(k_{\psi} d\right) /\left(k_{\psi} d\right)$, with region of significant gust responses shaded in gray.

From Eq. 17, the transition frequency $f_{\text {crit }}$ between the low- and high-frequency regions can be determined as where $k_{\psi}^{\text {crit }}=2 \pi / d$, which simplifies to

$$
f_{\text {crit }}=\frac{c_{0} \beta}{d}
$$




\section{III.C. Planar Shear Layer Refraction Model}

The analysis made so far considers the airfoil is completely surrounded by a uniformly convecting medium, such as in a closed-jet wind tunnel. However, open-jet wind tunnel setups are also very common for aeroacoustic measurements. In such cases, a shear layer will develop in the interface between the convected and the steady regions, and sound waves propagating through this shear layer will be refracted, affecting both their amplitude and travel time. Both effects must then be incorporated into the propagation model used to estimate the sound radiation by the acoustic sources. Similarly, knowledge of these effects allow them to be taken into account when applying backpropagation methods, such as beamforming. ${ }^{25}$

An open-jet wind tunnel with a rectangular nozzle was used for the experiments described in Section V, and is represented in Figure 5. The convecting region is represented in the lower part of the image, and the air in this region is being convected in the $+x$ direction at a velocity $U_{x}$. A point source is located inside the flow at $\mathbf{r}_{s}=\left(x_{s}, y_{s}, z_{s}\right)$, and a microphone is located outside the flow at $\mathbf{r}_{m}=\left(x_{m}, y_{m}, z_{m}\right)$. The shear layer is assumed to be a plane surface of zero thickness, and is represented as a plane at height $z=z_{l}$ and indicated by the dotted line. It is implicitly required that $z_{l}$ must be located between $z_{s}$ and $z_{m}$.

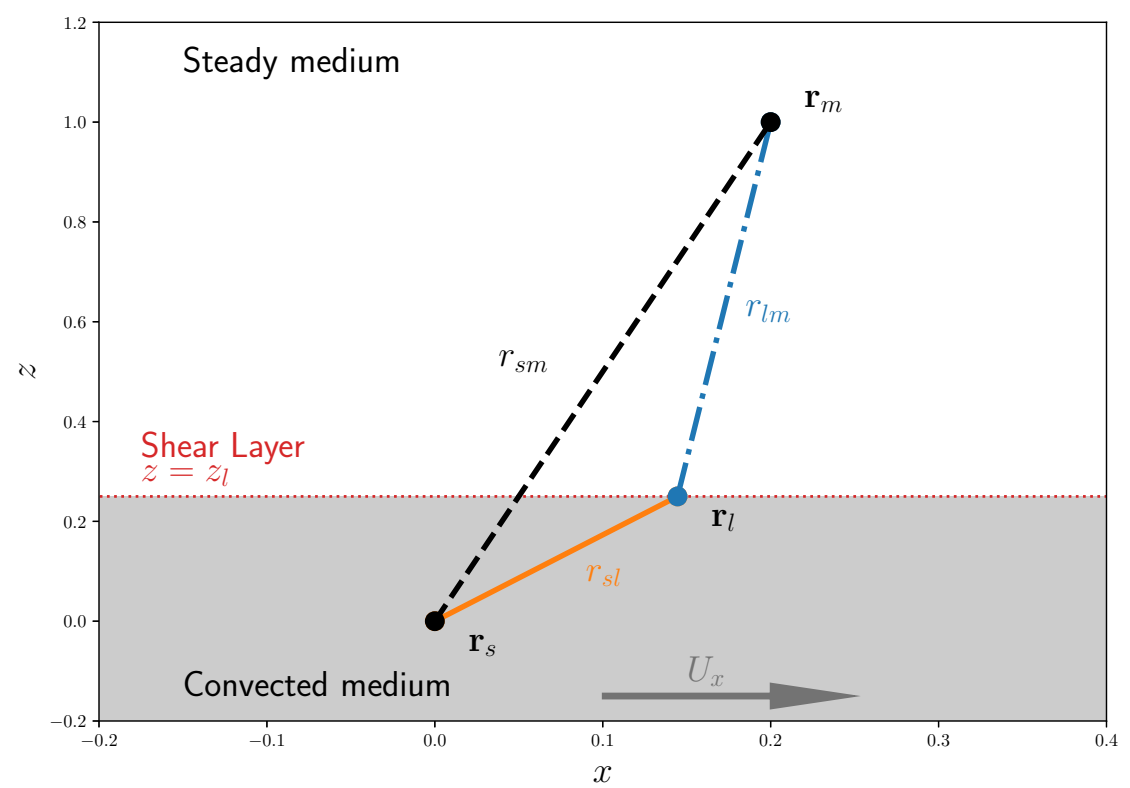

Figure 5: Cross-section in the $x z$ plane of an acoustic ray trajectory across a shear layer.

An acoustic ray is emitted by the source and crosses the shear layer at the point $\mathbf{r}_{l}=\left(x_{l}, y_{l}, z_{l}\right)$, where it is refracted and changes direction, before reaching the microphone at $\mathbf{r}_{m}$. The path $r_{s l}$ occurs inside the flow, and hence must obey the convected wave equation; meanwhile, the path $r_{l m}$ occurs in the quiescent medium region, and thus must obey the standard wave equation. There are now two variables that must be determined: the total propagation time $\tau_{s m}$ taken by the acoustic ray to travel from the source through the shear layer and to the microphone, and the intermediate propagation distances $r_{s l}$ and $r_{l m}$ covered by the acoustic ray before and after crossing the shear layer, respectively.

A simplified analytical model for describing the propagation time and amplitude decay of an acoustic ray moving through a shear layer is proposed. A similar approach has also been adopted by Bahr et al. ${ }^{26}$ Although not discussed here, Amiet's model for shear layer correction ${ }^{27}$ yields identical results to the proposed method.

\section{III.C.1. Total Propagation Time}

The Fermat's Principle of least travel time can be used to determine the shear layer crossing point $\mathbf{r}_{l}$ : this point will be such that minimizes the total propagation time $\tau_{s m}$ from source to microphone. ${ }^{28,29}$

We define a convection-corrected distance $\sigma_{s l}$ between the source point $\mathbf{r}_{s}$ and the shear layer crossing point $\mathbf{r}_{l}$ as 


$$
\sigma_{s l}=\sqrt{\left(x_{l}-x_{s}\right)^{2}+\beta^{2}\left[\left(y_{l}-y_{s}\right)^{2}+\left(z_{l}-z_{s}\right)^{2}\right]} .
$$

The time $\tau_{s l}$ taken for the ray to propagate from $\mathbf{r}_{s}$ to $\mathbf{r}_{l}$ can be obtained from the convected wave equation as ${ }^{25}$

$$
\tau_{s l}=\frac{-\left(x_{l}-x_{s}\right) M_{x}+\sigma_{s l}}{c_{0} \beta^{2}}
$$

Outside the mean flow, the acoustic ray must obey the standard wave equation and move with the speed of sound $c_{0}$ in any direction. The time $\tau_{l m}$ taken for the ray to propagate from the shear layer crossing point $\mathbf{r}_{l}$ to the microphone $\mathbf{r}_{m}$ is written as

$$
\tau_{l m}=\frac{r_{l m}}{c_{0}}
$$

and the total propagation time is given by the sum of the two intermediate times:

$$
\tau_{s m}=\tau_{s l}+\tau_{l m}
$$

Taking the partial derivatives of Equation 26 with respect to the shear layer coordinates $x_{l}$ and $y_{l}$ leads to the following system of equations: ${ }^{26-28}$

$$
\left\{\begin{array}{l}
\left(\frac{x_{l}-x_{s}}{\sigma_{s l}}\right)-\beta^{2}\left(\frac{x_{m}-x_{l}}{r_{l m}}\right)-M_{x}=0, \\
\left(\frac{y_{l}-y_{s}}{\sigma_{s l}}\right)-\left(\frac{y_{m}-y_{l}}{r_{l m}}\right)=0 .
\end{array}\right.
$$

These equations present constraints on the shear layer crossing point in the $x$ and $y$ directions, and the $z_{l}$ coordinate is already constrained to be on the shear layer plane.

It is now possible to numerically calculate the shear layer crossing point $\mathbf{r}_{l}$ that minimises the acoustic ray total travel time (Eq. 26), subject to the constraints given by Eq. 27. The minimization routine scipy.optmize.minimize from the SciPy package ${ }^{30}$ is used for this estimation, with an initial guess given by the point where a straight line between the source and the observer intersects the shear layer plane. Once the point $\mathbf{r}_{l}$ is found, the total propagation time is determined from Equation 26.

The shear layer crossing point $\mathbf{r}_{l}$ and total propagation time $\tau_{s m}$ are frequency-independent, and can be precalculated and stored for all pairs $\left(\mathbf{r} \mid \mathbf{r}_{s}\right)$ of observer locations and airfoil surface points in a given test setup configuration.

\section{III.C.2. Amplitude Decay across a Shear Layer for a Dipole Source}

Equation 5 for a point dipole in a moving medium can be used to describe an acoustic ray propagating from a point dipole source towards the shear layer crossing point. This expression can be rewritten as the product of an amplitude term and a complex exponential term containing the acoustic ray phase, shown below separated by square brackets:

$$
\frac{\partial}{\partial z_{s}} G_{U_{x}}\left(\mathbf{r}_{l} \mid \mathbf{r}_{s}, \omega\right)=\left[\left(j k_{0}+\frac{1}{\bar{r}}\right)\left(\frac{z_{l}-z_{s}}{\sigma_{s l}}\right)\left(\frac{1}{4 \pi \sigma_{s l}}\right)\right]\left[e^{-j \omega \tau_{s l}}\right] .
$$

The acoustic ray amplitude in Eq. 28 contains three terms (separated by parenthesis), all evaluated over flowtransformed variables: the first term contains the dipole hydrodynamic near field, the second contains the dipole directivity, and the third contains the spherical amplitude decay with distance.

It is now assumed that the the dipole near-field and the spherical spreading amplitude decay will continue to affect the acoustic ray amplitude after the ray crosses the shear layer, and must be evaluated over non-transformed variables beyond the shear layer. The dipole directivity, on the other hand, must be a property inherent to the angle of emission of the acoustic ray when radiated from the source and cease to affect it once the ray has been emitted, and hence cannot depend on the total propagation distance. The expression for the dipole field across a shear layer adopted here is

$$
\frac{\partial}{\partial z_{s}} G_{U_{x}}\left(\mathbf{r}_{s} \mid \mathbf{r}_{m}, \omega\right)=\left(j k_{0}+\frac{1}{\bar{r}_{s l}+r_{l m}}\right) \frac{\left(z_{l}-z_{s}\right)}{\sigma_{s l}} \frac{e^{-j \omega\left(\tau_{s l}+\tau_{l m}\right)}}{4 \pi\left(\sigma_{s l}+r_{l m}\right)} .
$$


Once the shear layer crossing points $\mathbf{r}_{l}$ and total propagation times $\tau_{s m}$ are obtained for every pair $\left(\mathbf{r} \mid \mathbf{r}_{s}\right)$ of airfoil and observer points as outlined above, Equation 29 can be used to include the shear layer refraction effects in the transfer function between these points.

\section{Surface Pressure Prediction}

This section presents predictions of single-gust flat plate surface pressure characteristics obtained with the Amiet model. This physical quantity is difficult to measure in practice, as a real airfoil immersed in a turbulent flow will develop not only the acoustic unsteady pressure jump but also a significantly larger wall pressure fluctuation due to the turbulent boundary layer. ${ }^{31}$ Hence, these predictions provide important information about the acoustic behaviour of the airfoil that are very difficult to obtain experimentally.

Figure 6 shows examples of single-gust response functions for a range of supercritical and subcritical gusts at the normalised frequency $k_{0} c=5$ and low Mach number $M_{x} \approx 0.17$, with Figure 6a showing the response function chordwise magnitude, and Figure $6 \mathrm{~b}$ showing the surface pressure jump over the entire airfoil surface.

The $\left(x_{s}^{-1 / 2}\right)$-type singularity at the leading edge is visible for all gusts. Supercritical gusts can be seen to load more significantly the airfoil aft sections, while subcritical gusts are more concentrated near the leading edge and decay exponentially towards the trailing edge. It can also be seen how the airfoil response wavefronts move from towards the downstream direction for a parallel-incidence gust, to towards the spanwise direction for oblique gusts.

\section{Acoustic Radiation Prediction and Experimental Validation}

We now present the acoustic radiation predictions using the complete model (Eq. 8), and validate them against experimental measurements made with a compact microphone array parallel to the airfoil. All cross-spectral densities herein are presented as single-sided and as a function of the temporal frequency $f$ (instead of the angular frequency $\omega=2 \pi f$ ), hence an extra multiplying factor of $4 \pi$ is included in the calculations.

The measurements were performed in the ISVR Large Anechoic Chamber using the DARP Open Jet Facility, ${ }^{32}$ a low-noise, low-turbulence wind tunnel, with its working section located inside the ISVR Large Anechoic Chamber. A diagram of the experimental setup is shown in Figure 7: a flat-plate airfoil is situated in a turbulent flow, and its radiated acoustic pressure is measured with a planar microphone array positioned below the airfoil. The wind tunnel nozzle has dimensions of $0.15 \times 0.45 \mathrm{~m}$, and holds two sideplates used to fix the airfoil within the mean flow. Due to the nozzle dimensions, a shear layer will develop at $z_{s l}=-0.075 \mathrm{~m}$ - i.e. below the airfoil.

Airfoil noise measurements were performed using a flow speed of $U_{x}=60 \mathrm{~m} / \mathrm{s}$. Turbulent flows can be achieved by introducing a turbulence-generating grid in the nozzle, and the turbulence intensity and integral length scale were measured using hot-wire anemometry and found to be $T_{i}=0.025$ and $\Lambda=0.007 \mathrm{~m}$, respectively. The turbulence wavenumber spectrum for the noise predictions was modelled using the von Karman model for isotropic turbulence (Eq. 18) using the above mentioned flow parameters.

\section{V.A. Planar Microphone Array Setup}

The planar microphone array was positioned at approximately $0.5 \mathrm{~m}$ below the airfoil, with the center microphone visually aligned with the airfoil mid span, as depicted in Fig. 7.

The array was built on a $60 \times 60 \mathrm{~cm}, 1 \mathrm{~cm}$-thick plywood board, using electret condenser capsules mounted flush to the plywood board surface. The capsules were soldered to their associated polarizing circuit, and connected to a custom-made multi-channel microphone amplifier using coaxial cables. The amplifier outputs were then connected to a rack of analog-to-digital converters, which were connected to a laptop via a USB interface. The chosen sampling frequency was $48 \mathrm{kHz}$, and each recording lasted for $30 \mathrm{~s}$.

The planar array microphone signal amplitudes were later calibrated using a $1 \mathrm{kHz}, 1 \mathrm{~Pa}$ RMS reference signal from a B\&K Type 4230 calibrator positioned above each microphone, from which a calibration gain is derived for each array channel and later applied to the raw microphone data.

The cross-spectral characteristics of the measured acoustic pressures are compared to the predicted characteristics for different microphones. We choose to investigate the microphones numbered 32 to 35 , which are positioned at increasing spanwise coordinate $y$, as shown in Figure 8. These can be used to compare the predicted and measured spanwise behaviour of the radiated acoustic field, as previously mentioned. We investigate their power spectral density magnitude, as well as the cross-spectral density phase and coherence-squared function - the latter two using the center microphone (number 01) as the phase reference. 


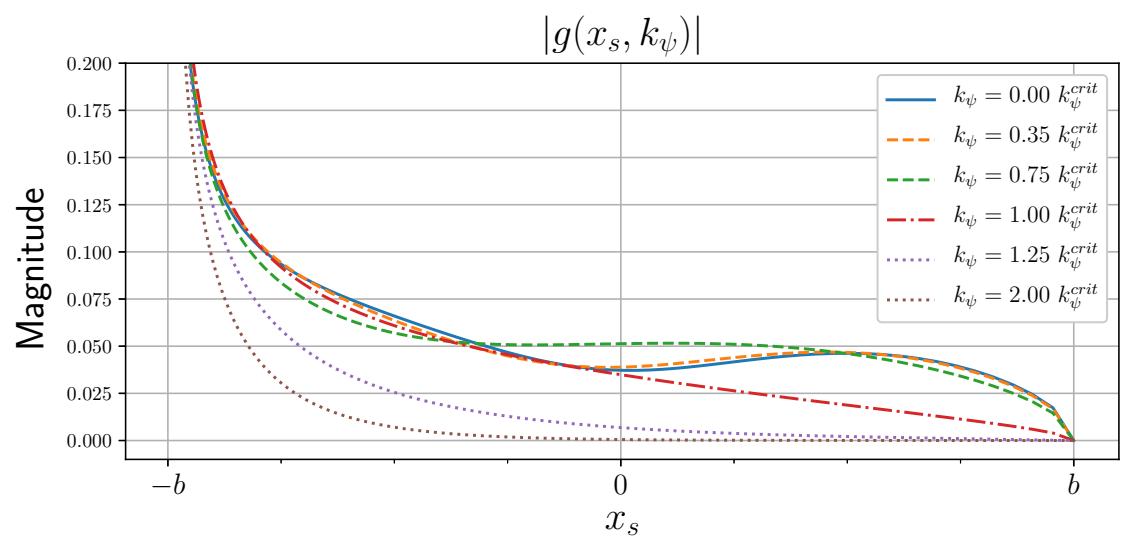

(a)

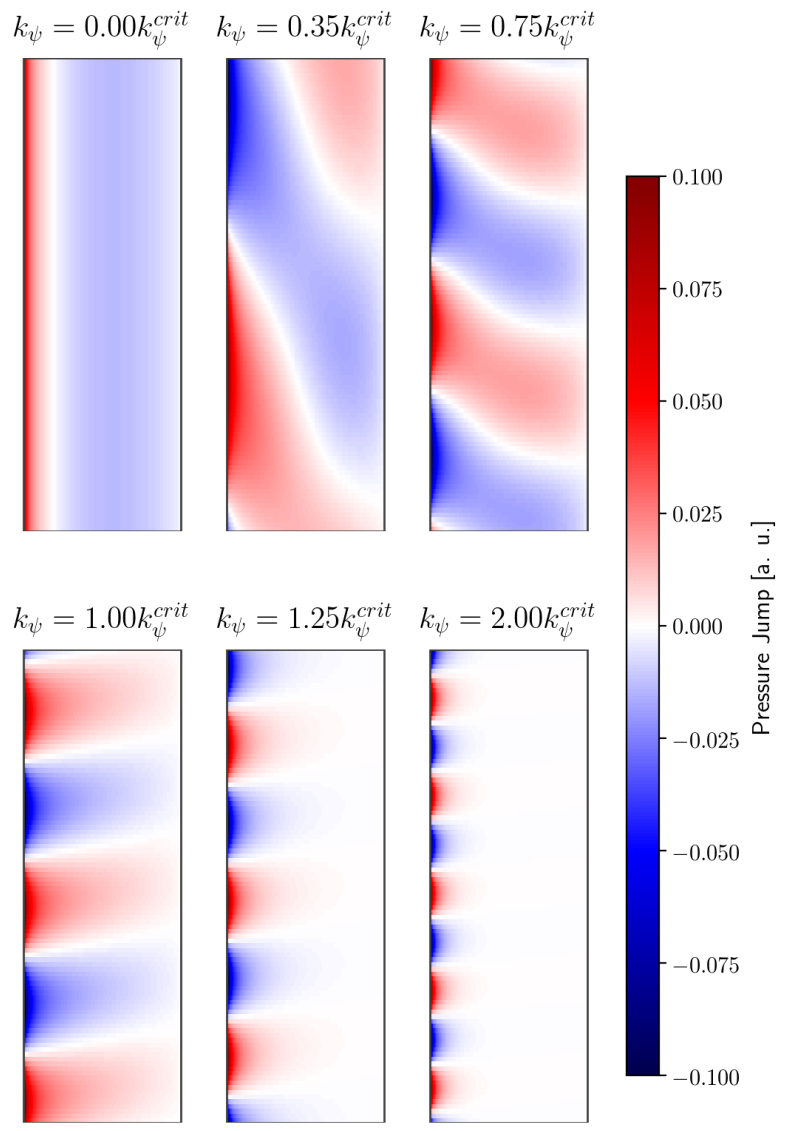

(b)

Figure 6: Single-gust airfoil response function for different gusts with $k_{0} c=5, M_{x} \approx 0.17$. (a) chordwise magnitude; (b) real part of surface pressure jump. 


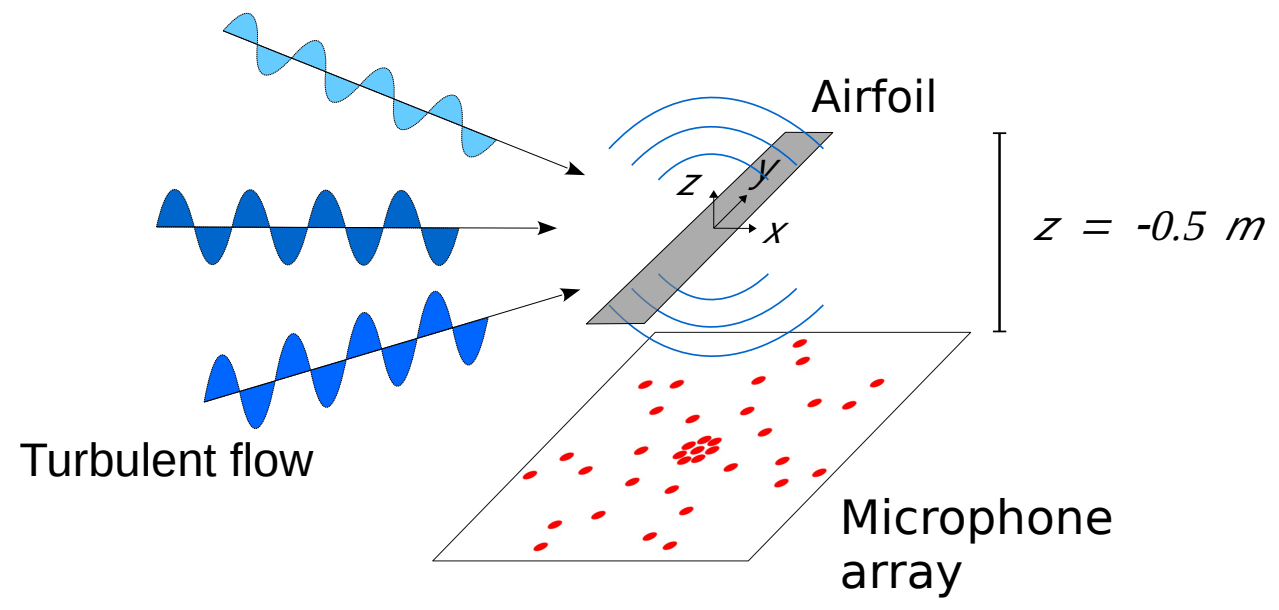

Figure 7: Experimental setup for turbulence-airfoil interaction noise measurements with a planar microphone array.

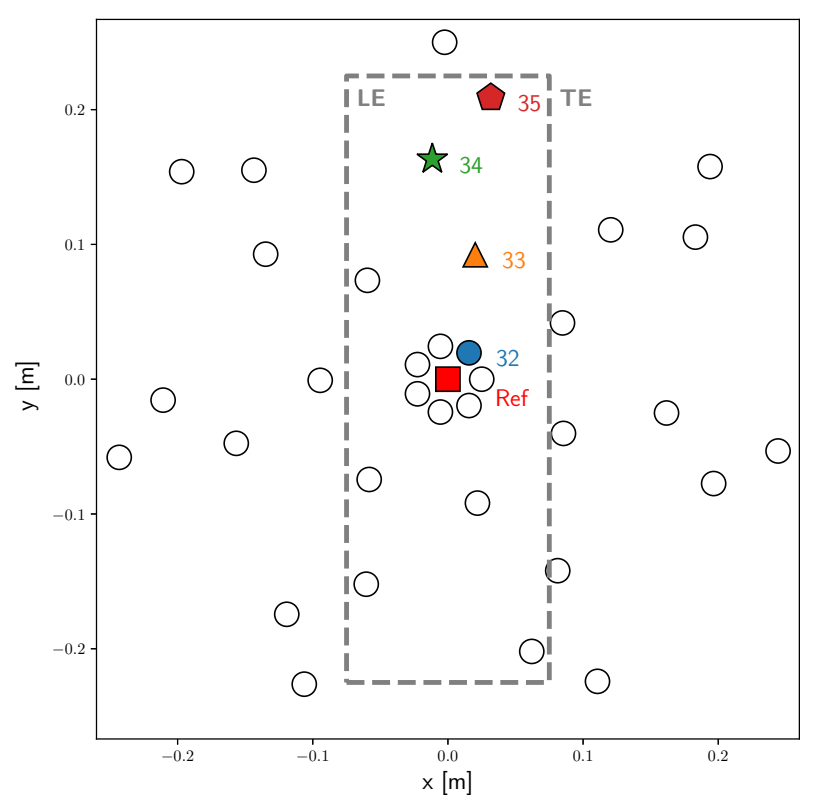

Figure 8: Top-down view of multiarm spiral array, with selected microphones for spanwise ( $y$ direction) acoustic field analysis. The airfoil position (above the array) is indicated with the thick, dashed lines, and the mean flow direction is from left to right. 
Figure 9 show a comparison of the predicted and measured PSD in $\mathrm{dB}$ re $(20 \mu \mathrm{Pa})^{2} / \mathrm{Hz}$ (top), unwrapped CSD phase (in radians, middle) and coherence-squared function $\gamma^{2}$ (bottom) for the selected planar array microphones, ordered in increasing spanwise distance $y$ from the array centre. The cross-spectra were calculated from the calibrated microphone signals using Welch's method, ${ }^{33}$ using $N_{D F T}=1024$ samples, $50 \%$ overlap and a Hann window.

\section{V.B. PSD Levels}

The spectra levels display slight oscillations in all measured data, in a manner similar to a "comb filter" effect, with peaks at approximately every $340 \mathrm{~Hz}$. This was later identified as a standing wave effect between the flat plate airfoil and the planar microphone array baffle.

All power spectral density plots in Figures 9a to 9d show significant differences between the predictions and the measurements in both level and general spectral shape, with differences of more than $10 \mathrm{~dB}$ are visible over a wide range of frequencies in all plots. The rate of decay is also different between the predictions and measurements. However, some oscillation features in the analytical model are weakly visible in the measurements, such as the notch at approximately $2.5 \mathrm{kHz}$.

Amongst possible reasons for these discrepancies are the wind tunnel jet noise, which is particularly prominent at low frequencies. Similarly, the planar array is relatively close to the wind tunnel shear layer, which might also contaminate the measurements with hydrodynamic near-field noise at those same frequencies. Previous analyses of this experimental data using beamforming methods ${ }^{21}$ have identified the presence of parasitic corner sources, ${ }^{34,35}$ which can also interfere with the acoustic measurements; however, the extent of this interference in the measured PSDs has yet to be determined.

\section{V.C. CSD Phase and Coherence}

Figures $9 \mathrm{a}$ to $9 \mathrm{~d}$ show the unwrapped cross-spectrum phase and the coherence of microphones 32 (closest to the array center) to 35 (further from the array center), relative to microphone 01 at the array center. These plots have a lowfrequency zone where the coherence rolls off from approximately unity to zero, and a high frequency zone where the coherence remains very close to zero, with some minor oscillations.

At low frequencies, the airfoil response to single gusts will exhibit an almost omnidirectional radiation (up to a factor proportional to the dipole directivity), and every gust will induce a sound field that is fully coherent across all spanwise observers, as observed in both predictions and measurements ${ }^{\mathrm{a}}$. The comb-filtering effect observed in the PSDs is not strongly present in this frequency range, possibly because the array baffle is not large enough (compared to the acoustic wavelength) to be a significant reflector of sound. In general, the phase and coherence predictions agree well within this region.

The airfoil radiation becomes more directive at higher frequencies, inducing each spanwise microphone to be "illuminated" by the radiation from a single gust. The noise observed by each microphone becomes uncorrelated to the noise observed by the reference (microphone 01), leading to the coherence roll-off. There is still a good agreement between the predicted and the measured phase and coherence trends, with larger differences visible where the coherence curves drop to zero. The comb-filtering effects also become more prominent in this zone, possibly because the array baffle dimensions become comparable to the acoustic wavelength. As incoherent signals by definition do not have a well-defined phase difference, the measured phase difference diverges significantly from the predictions at even higher frequencies (not visible in all plots).

\section{V.D. Coherence Between all Microphones}

We now consider how the acoustic field coherence varies as a function of frequency over all array microphones. For this analysis, we calculate the pairwise coherence for each microphone referenced to microphone 01, and plot the coherence over the array surface at select narrowband frequencies. For visualization purposes, the pairwise coherence function is linearly interpolated between the sensor pairs.

Figure 10 show the predicted (left) and measured (right) coherence function for narrowband frequencies close to $200 \mathrm{~Hz}$ (top), $1 \mathrm{kHz}$ (middle) and $4 \mathrm{kHz}$ (bottom), with microphone 1 at the centre of the array (marked with a blue square) as the phase reference. The previously discusse coherence behaviour of the radiated sound field is clearly visible in these plots: all microphones are highly coherent at low frequencies, and the spanwise coherence decreases with increasing frequency, as the airfoil single-gust response begins to become more directional. Since the chordwise

${ }^{a}$ The exception is microphone 32, whose measured coherence drops at very low frequencies due to interference from $100 \mathrm{~Hz}$ electrical noise. 

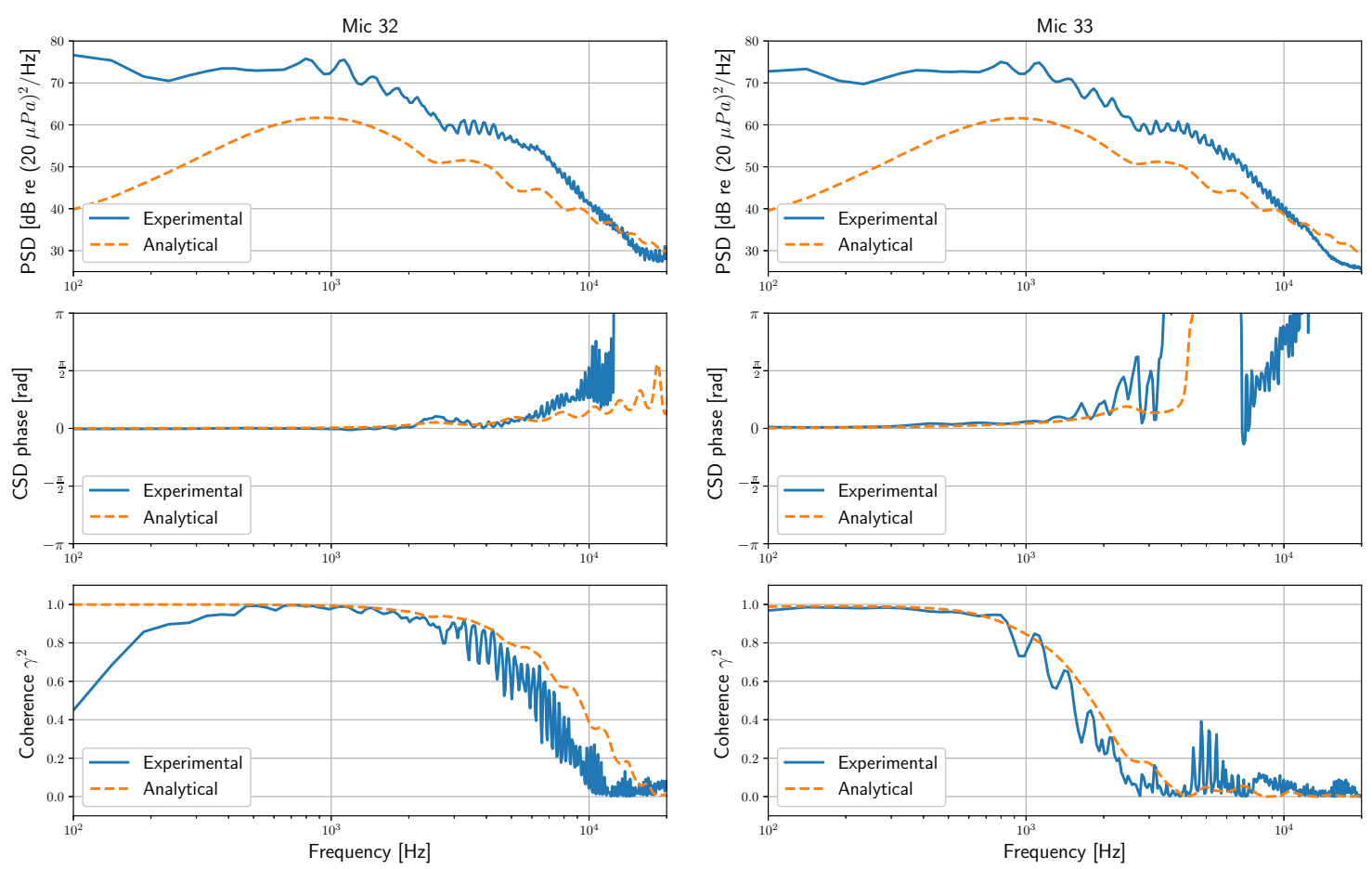

(a)
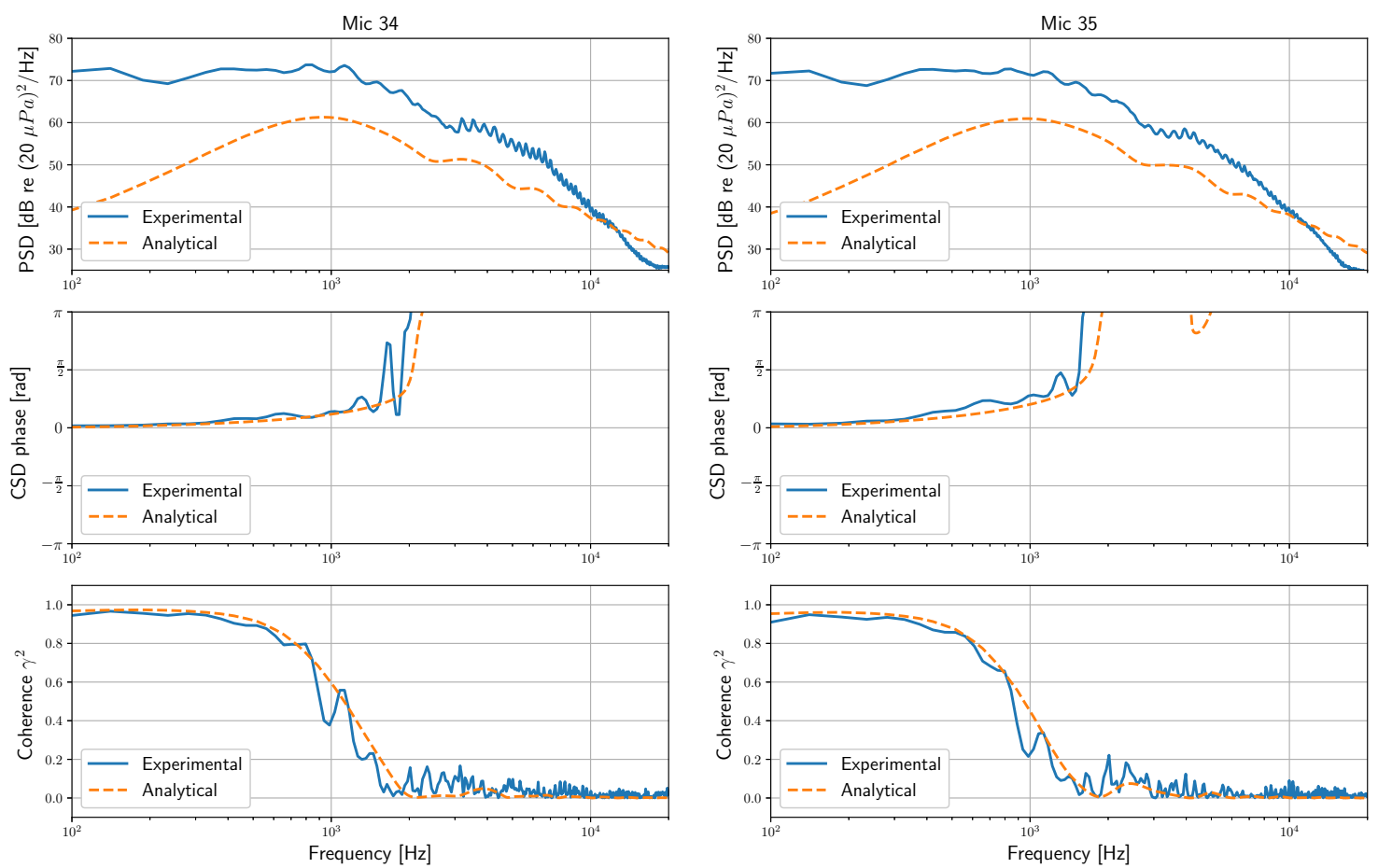

(c)

(d)

Figure 9: Analytical (dashed lines) and experimental (solid lines) PSD magnitudes (top, in $\mathrm{dB}$ re $(20 \mu \mathrm{Pa})^{2} / \mathrm{Hz}$ ), unwrapped CSD phase (middle, in radians) and coherence $\gamma^{2}$ (bottom, ref mic 01) as a function of frequency for different microphones: (a) mic 32; (b) mic 33; (c) mic 34; (d) mic 35. 
radiation is always fully coherent, the coherence is preserved over a larger range of frequencies for microphones that are aligned in the chordwise direction.

\section{Conclusions}

An open implementation of the Amiet model for leading edge noise is described. This model includes the classical formulation using far-field and infinite-span (i.e. single-gust) approximation, and also the less common formulation including finite-span, subcritical gusts and near-field effects, allowing for more general use. Functions are provided to calculate both the surface pressure and the radiated noise cross-spectral densities, and some implementation details are described. Good agreement between predictions and experimental measurements are obtained, especially regarding features in the cross-spectrum phase - an important variable for simulating phased array measurements, for example. PSD levels show some differentes between predictions and measurements, which are hypothesized to be due to increased wind tunnel and shear layer noise at the relatively short measurement distance adopted here.

For simplicity, this implementation does not adopt significant optimizations for computational speed, and as a consequence can be fairly slow at higher frequencies, where many turbulent gusts are acoustically significant. It also does not add any form of background noise to the acoustic field measurements, since noise levels are very contextdependent and can be added to the cross-spectra at a later stage.

The codes in Python language can be obtained from Github via the following link:

- https://github.com/fchirono/amiet_tools

A collection of test scripts is provided with the source code, containing examples of how to calculate the surface pressure and radiated sound field for single-gust, multiple-gusts, surface pressure cross-spectral density analysis, and beamforming simulation. Users are invited to download the codes and sample test scripts, and to collaborate with the project development via Github or by contacting the first author directly. Sample microphone array cross-spectral matrices (CSMs) are also provided for the test setup discussed here, containing the CSMs obtained via analytical predictions and experimental measurements and used to generate the results in Section V.

The next steps include developing an optional feature to incorporate the aforementioned corner sources at the airfoil leading edge to the acoustic field CSD prediction. An extension to the current package to include trailing-edge noise is also currently being investigated.

\section{Acknowledgments}

The Authors would like to thank Dr. Chaitanya Paruchuri for his help with the experiments and insightful discussions. Fabio Casagrande Hirono would like to thank the National Council for Scientific and Technical Development (Conselho Nacional de Desenvolvimento Científico e Tecnológico - CNPq) of the Federal Government of Brazil for sponsoring his $\mathrm{PhD}$ degree under the "Science Without Borders" program. 

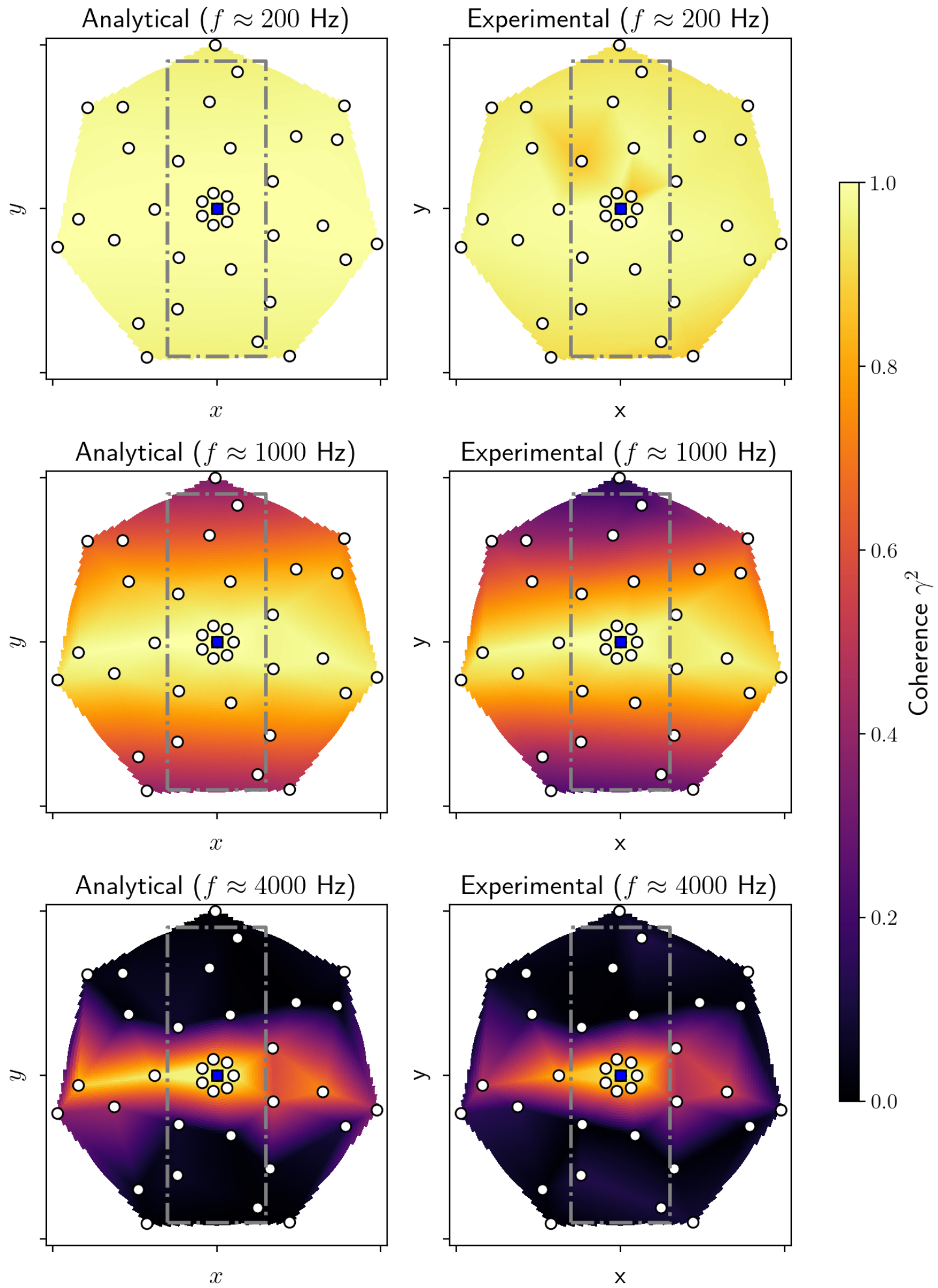

Figure 10: Analytical (left) and experimental (right) coherence function $\gamma^{2}$ evaluated over the microphone array face with reference to microphone 1 (array centre, square marker). Values are linearly interpolated from pairwise coherences at approximate narrowband frequencies $200 \mathrm{~Hz}$ (top), $1 \mathrm{kHz}$ (middle) and $4 \mathrm{kHz}$ (bottom). 


\section{A. Appendix A: Algorithm Pseudocode}

The pseudocode used to evaluate the airfoil surface pressure cross-spectral density $S_{\Delta p \Delta p^{\prime}}$ and/or the radiated sound cross-spectral density $S_{p p^{\prime}}$ is shown below. Most of the instructions displayed here have equivalent Python functions or methods associated to it in the package, and do not need to be manually implemented. For more details on how to use these functions, see the documentation and example scripts included with the package.

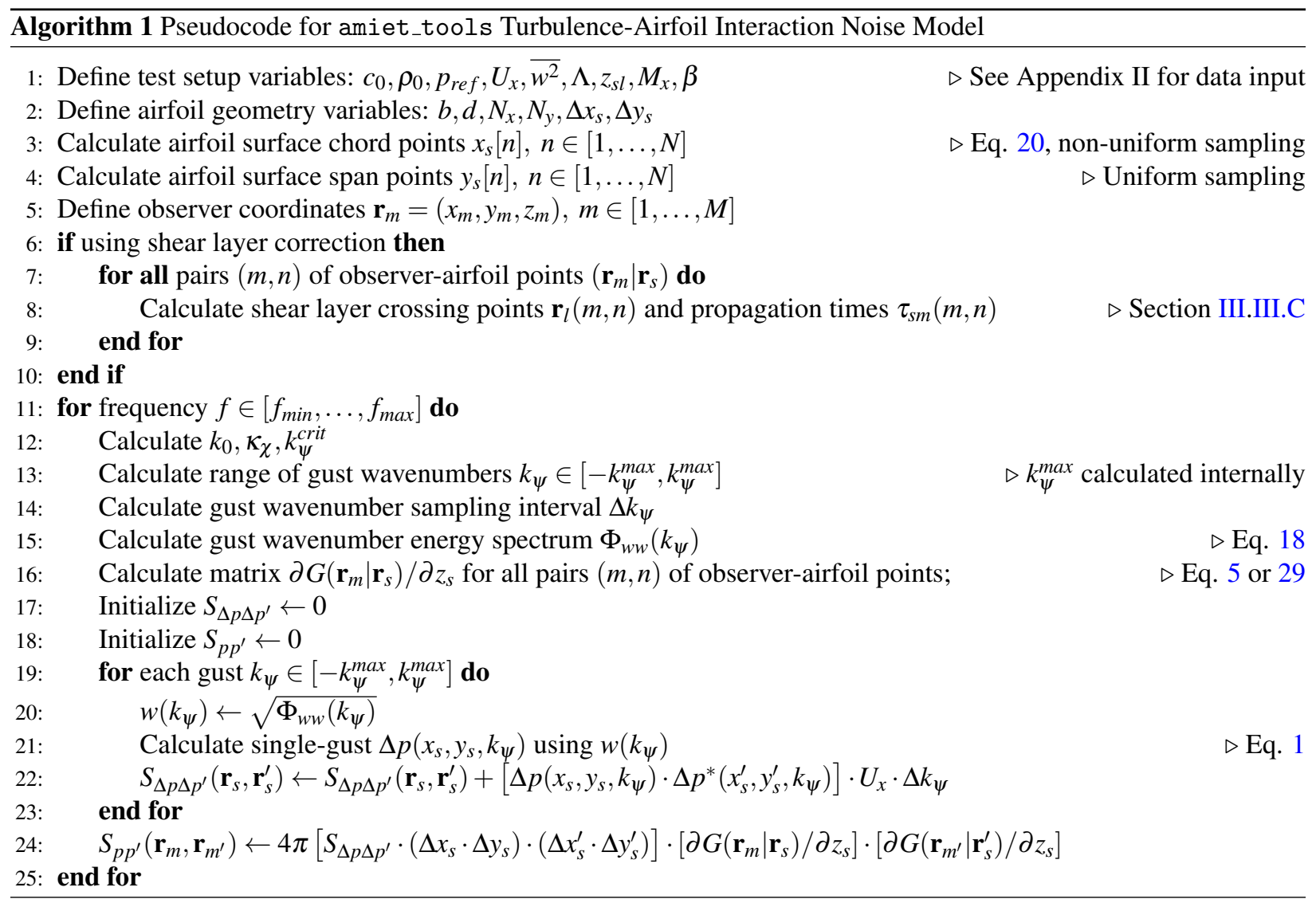




\section{B. Appendix B: Data Input Using amiet_tools Classes}

The amiet_tools package uses Python classes - i.e. object-oriented constructs - to store data related to the different aspects of the simulation. The three classes are described below, together with a listing of the attributes they contain:

- Class TestSetup:

- TestSetup.c0: speed of sound in a steady medium $c_{0}($ in $\mathrm{m} / \mathrm{s})$;

- TestSetup.rho0: air density $\rho_{0}\left(\right.$ in $\mathrm{kg} / \mathrm{m}^{3}$ );

- TestSetup.p_ref: reference acoustic pressure $p_{\text {ref }}=20 \mu \mathrm{Pa}$ RMS;

- TestSetup. Ux: mean flow velocity $U_{x}$ (in $\mathrm{m} / \mathrm{s}$ );

- TestSetup.turb_intensity: turbulence intensity $\overline{w^{2}} / U_{x}$;

- TestSetup.length_scale: turbulence length scale $\Lambda$ (in $\mathrm{m}$ );

- TestSetup.z_sl: shear layer height $z_{s l}$ (in m);

- TestSetup.flow_dir: single character denoting direction of the mean flow (i.e. ' $\mathrm{x}$ ' to denote flow in $+x$ direction);

- TestSetup.dipole_axis: single character denoting direction of dipoles' axes (i.e. 'z' for dipoles pointing "up", in $+z$ direction);

- TestSetup.Mach: mean flow Mach number $M_{x}=U_{x} / c_{0}$;

- TestSetup. beta: Prandtl-Glauert factor $\beta=\sqrt{1-M_{x}^{2}}$.

- Class AirfoilGeom:

- AirfoilGeom.b: airfoil semichord $b=c / 2$ (in m);

- AirfoilGeom.d: airfoil semispan $d=L / 2$ (in $\mathrm{m}$ );

- AirfoilGeom.Nx: number of chordwise points $N_{x}$, non-uniform sampling;

- AirfoilGeom. Ny: number of chordwise points $N_{y}$, uniform sampling;

- AirfoilGeom.dx: airfoil surface chordwise sampling intervals $\Delta x_{s}$ (generated internally in the code)

- AirfoilGeom.dy: airfoil surface spanwise sampling interval $\Delta y_{s}$ (generated internally in the code).

- Class FrequencyVariables:

- FrequencyVariables.freq: frequency $f$ (in $\mathrm{Hz}$ );

- FrequencyVariables.k0: acoustic wavenumber $k_{0}=2 \pi f / c_{0}$;

- FrequencyVariables.Kx: gust chordwise wavenumber $\kappa_{\chi}=\omega / U_{x}$;

- FrequencyVariables.Ky_crit: gust critical spanwise wavenumber $k_{\psi}^{\text {crit }}$.

One instance of each class is created to store the variable values related to a given experimental condition and to pass them to the different functions in the package. Numerical values can be assigned directly via a Python script, or they can be read from an external file using the provided functions loadTestSetup and loadAirfoilGeom. For more implementation details and how to use these functions, see the documentation and example scripts included with the package.

A fourth class, called MicArrayCsmHDF5, is also included in the package and allows the reading and writing of microphone array CSM data in the HDF5 file format adopted by the Array Methods community ${ }^{36,37}$ for sharing beamforming data. 


\section{References}

${ }^{1}$ Lyu, B. and Azarpeyvand, M., "On the noise prediction for serrated leading edges," Journal of Fluid Mechanics, Vol. 826, 2017, pp. $205-234$.

-2Mathews, J. and Peake, N., "An analytically-based method for predicting the noise generated by the interaction between turbulence and a serrated leading edge," Journal of Sound and Vibration, Vol. 422, 2018, pp. 506-525.

${ }^{3}$ Cannard, M., Joseph, P., Turner, J., Kim, J., and Chaitanya, P., "Physical mechanisms and performance of slitted leading-edge profiles for the reduction of broadband aerofoil interaction noise," Journal of Sound and Vibration, Vol. 473, 2020, pp. 1-17.

${ }^{4}$ Clair, V., Polacsek, C., Garrec, T. L., Reboul, G., Gruber, M., and Joseph, P., "Experimental and Numerical Investigation of TurbulenceAirfoil Noise Reduction Using Wavy Edges," AIAA Journal, Vol. 51, No. 11, 2013, pp. 2695-2713.

${ }^{5}$ Narayanan, S., Chaitanya, P., Haeri, S., Joseph, P., Kim, J., and Polacsek, C., "Airfoil noise reductions through leading edge serrations," Physics of Fluids, Vol. 27, No. 025109, 2015.

${ }^{6}$ Ayton, L., "Bioinspired aerofoil adaptations: the next steps for theoretical models," Phil. Trans. R. Soc. A, Vol. 377, 2019, pp. 1-14.

${ }^{7}$ Bampanis, G., Roger, M., Ragni, D., Avallone, F., and Teruna, C., "Airfoil-Turbulence Interaction Noise Source Identification and Reduction by Leading-Edge Serrations," 25th AIAA/CEAS Aeroacoustics Conference, 2019, AIAA Paper 2019-2741.

${ }^{8}$ Lyu, B., Ayton, L., and Chaitanya, P., "On the acoustic optimality of leading-edge serration profiles," Journal of Sound and Vibration, Vol. 462, 2019, pp. 1-17.

$\checkmark{ }^{9}$ Roger, M., "Broadband Noise from Lifting Surfaces: Analytical Modeling and Experimental Validation," Noise Sources in Turbulent Shear Flows: Fundamentals and Applications, edited by R. Camussi, Springer-Verlag Wien, 2013.

${ }^{10}$ Adamczyk, J., "The Passage of an Infinite Swept Airfoil Through an Oblique Gust," Tech. Rep. NASA Contractor Report CR-2395, National Aeronautics and Space Administration (NASA) - USA, 1974.

${ }^{11}$ Graham, J., "Similarity rules for thin aerofoils in non-stationary subsonic flows," Journal of Fluid Mechanics, Vol. 43, part 4, 1970, pp. 753766.

${ }^{12}$ Amiet, R. K., "Acoustic Radiation from an Airfoil in a Turbulent Stream," Journal of Sound and Vibration, Vol. 41, No. 4, 1975 , pp. 407-420.

${ }^{13}$ Santana, L. D., Schram, C., and Desmet, W., "Low-frequency extension of Amiet's theory for compact airfoil noise predictions," Journal of Sound and Vibration, Vol. 372, No. 23, 2016, pp. 342-356.

${ }^{14}$ Santana, L. D., Christophe, J., Schram, C., and Desmet, W., "A Rapid Distortion Theory modified turbulence spectra for semi-analytical airfoil noise prediction," Journal of Sound and Vibration, Vol. 383, 2016, pp. 349-363.

${ }^{15}$ Roger, M. and Moreau, S., "Extensions and limitations of analytical airfoil broadband noise models," International Journal of Aeroacoustics, Vol. 9, No. 3, 2010, pp. 273-305.

${ }^{16}$ Wilson, G., Aruliah, D. A., Brown, C. T., Chue Hong, N. P., Davis, M., Guy, R. T., Haddock, S. H. D., Huff, K. D., Mitchell, I. M., Plumbley, M. D., Waugh, B., White, E. P., and Wilson, P., "Best Practices for Scientific Computing," PLOS Biology, Vol. 12, No. 1, 01 2014, pp. 1-7.

${ }^{17}$ Sarradj, E. and Herold, G., "A Python framework for microphone array data processing," Applied Acoustics, Vol. 116, 2017, pp. 50-58.

${ }^{18}$ Wukie, N., Lindblad, D., and Andersson, N., "noisyduck: an open-source Python tool for computing eigenmode decompositions of duct flows," 25th AIAA/CEAS Aeroacoustics Conference, 2019, AIAA Paper 2019-2418.

${ }^{19}$ Reboul, G., Modélisation du bruit à large bande de soufflante de turboréacteur, Phd thesis, Laboratoire de Mécanique des Fluides et d'Acoustique - École Centrale de Lyon, Lyon - France, 2010.

${ }^{20}$ de Santana, L., Semi-analytical methodologies for airfoil noise prediction, Phd thesis, Faculty of Engineering Science - Katholieke Universiteit Leuven, Leuven - Belgium, 2015.

${ }^{21}$ Casagrande Hirono, F., Far-Field Microphone Array Techniques for Acoustic Characterisation of Aerofoils, Phd thesis, Institute of Sound and Vibration Research - University of Southampton, Southampton - UK, 2018.

${ }^{22}$ Roger, M., "Sound Radiation by Moving Surfaces and the Green's Functions Technique," Noise Sources in Turbulent Shear Flows: Fundamentals and Applications, edited by R. Camussi, Springer-Verlag Wien, 2013.

${ }^{23}$ Chapman, C. J., "Similarity Variables for Sound Radiation in a Uniform Flow," Journal of Sound and Vibration, Vol. 233, No. 1, 2000, pp. 157-164.

${ }^{24}$ Paterson, R. and Amiet, R., "Acoustic Radiation and Surface Pressure Characteristics of an Airfoil due to Incident Turbulence," Tech. Rep. NASA Contractor Report CR-2733, National Aeronautics and Space Administration (NASA) - USA, 1976.

${ }^{25}$ Sijtsma, P., "Phased array beamforming applied to wind tunnel and fly-over tests," Tech. Rep. NLR-TP-2010-549, National Aerospace Laboratory (NLR) - the Netherlands, 2010.

${ }^{26}$ Bahr, C., Zawodny, N., Yardibi, T., Liu, F., Wetzel, D., Bertolucci, B., and Cattafesta, L., "Shear Layer Correction Validation using a Non-Intrusive Acoustic Point Source," 16th AIAA/CEAS Aeroacoustics Conference, 2010, AIAA Paper 2010-3735.

${ }^{27}$ Amiet, R., "Refraction of Sound by a Shear Layer," Journal of Sound and Vibration, Vol. 58, part 4, 1978, pp. 467-482.

${ }^{28}$ Koop, L., Ehrenfried, K., and Kröber, S., "Investigation of the systematic phase mismatch in microphone-array analysis," 11th AIAA/CEAS Aeroacoustics Conference, 2005, AIAA Paper 2005-2962.

$\checkmark{ }^{29}$ Porteous, R., Geyer, T., Moreau, D., and Doolan, C., "A correction method for acoustic source localisation in convex shear layer geometries," Applied Acoustics, Vol. 130, 2018, pp. 128-132.

${ }^{30}$ Jones, E., Oliphant, T., Peterson, P., et al., "SciPy: Open source scientific tools for Python,” Available online: http://www.scipy.org/, 2001, Accessed 23 April 2020.

${ }^{31}$ Bull, M., "Wall-pressure fluctuations beneath turbulent boundary layers: some reflections on forty years of research," Journal of Sound and Vibration, Vol. 190, 1996, pp. 299-315.

$\checkmark{ }^{32}$ Chong, T. P., Joseph, P., and Davies, P., "Design and performance of an open jet wind tunnel for aero-acoustic measurement," Applied Acoustics, Vol. 70, 2009, pp. 605-614.

${ }^{33}$ Shin, K. and Hammond, J., Fundamentals of Signal Processing for Sound and Vibration Engineers, John Wiley and Sons, Chichester, UK, 2008.

${ }^{34}$ Tuinstra, M. and Sijtsma, P., "Supression of spurious noise sources in airfoil self-noise measurements," 21 st AIAA/CEAS Aeroacoustics Conference, 2015, AIAA Paper 2015-2689. 
${ }^{35}$ Merino-Martinez, R., Sijtsma, P., and Snellen, M., "Inverse Integration Method for Distributed Sound Sources," 8th Berlin Beamforming Conference, 2018, BeBeC Paper BeBeC-2018-S07.

${ }^{36}$ Bahr, C., Jr., W. H., Ernst, D., Ahlefeldt, T., Spehr, C., Pereira, A., Leclere, Q., Picard, C., Porteous, R., Moreaux, D., Fischer, J., and Doolan, C., "A Comparison of Microphone Phased Array Methods Applied to the Study of Airframe Noise in Wind Tunnel Testing," 23rd AIAA/CEAS Aeroacoustics Conference, 2017, AIAA Paper AIAA 2017-3718.

${ }^{37}$ Sarradj, E., Herold, G., Sijtsma, P., Merino-Martinez, R., Malgoezar, A., Snellen, M., Geyer, T., Bahr, C., Porteous, R., Moreau, D., and Doolan, C., "A microphone array method benchmarking exercise using synthesized input data," 23rd AIAA/CEAS Aeroacoustics Conference, 2017, AIAA Paper AIAA 2017-3719. 